\title{
The Impact of Pharmacist-delivered Motivational Interviewing on Chronic Kidney Disease Identification and Management in Patients with Diabetes Mellitus and Low Socioeconomic Status
}

Keri DePatis, PharmD; Catherine Harrington, PharmD, PhD

Palm Beach Atlantic University, Gregory School of Pharmacy

\begin{abstract}
Purpose: Chronic kidney disease (CKD) is a common complication among patients with diabetes mellitus; however, noncompliance with the recommended annual screening is common. Increased screening among high-risk patients is important to identify the early stages $C K D$, potentially resulting in earlier treatment, slower progression, fewer complications, and decreased healthcare expenditures. Motivational interviewing (MI) has previously been shown to be effective for various behaviors, such as smoking cessation and cholesterol level control. The objective of this study is to evaluate the effectiveness of pharmacist-delivered MI compared to typical education (TE) methods in increasing CKD screening and subsequent angiotensin-converting enzyme inhibitor (ACE-I) or angiotensin II receptor blocker (ARB) initiation in high-risk patient populations.
\end{abstract}

Methods: Pharmacists screened diabetic patients within their chronic disease management clinic to identify patients that are at highrisk for CKD, indicated by a score of 4 or greater on the validated SCORED screening tool. High-risk patients were randomized to one of four groups to receive either one or two face-to-face education sessions from a pharmacist or student pharmacist using either MI or TE methods. Patients were then given the option to have their urine tested with a dipstick to detect albumin and creatinine, provided at no cost. The primary outcome was to determine the rate of urinary albumin testing, and the secondary outcome was to determine the rate of ACE-I or ARB initiation in patients found to have albuminuria.

Results: There were no significant differences in the rates of urinary albumin screening (87\% in TE vs. $100 \%$ in MI, $P=0.4828$ ) or subsequent $A C E-I / A R B$ initiation (100\% in TE and $50 \%$ in $\mathrm{MI}, P=1.000$ ) between education groups. Of the high-risk patients who underwent urinary albumin screening, $54 \%(n=15)$ were found to have proteinuria

Conclusions: While it appears that $\mathrm{MI}$ does not impact patient acceptance rates of microalbuminuria screening and ACE-I/ARB initiation, this study demonstrates the feasibility of pharmacist-delivered microalbuminuria screening in patients at high-risk for CKD in the outpatient setting.

Keywords: Chronic Kidney Disease, Ambulatory Care, Pharmacist, Diabetes, Screening, Microalbuminuria, Nephropathy

\section{Introduction}

Diabetic nephropathy is the primary cause of renal disease in the United States, occurring in $36 \%$ of patients with diabetes mellitus. ${ }^{1,2}$ Current guidelines recommend assessment of urinary albumin and estimated glomerular filtration rate at least annually in patients with type 1 diabetes with duration of 5 or more years, type 2 diabetes, or those with comorbid hypertension. ${ }^{3}$ However, previous studies indicate that a majority of patients with diabetes mellitus are not routinely screened for microalbuminuria, particularly those with low health literacy and socioeconomic status. ${ }^{4,5}$ Additional risk factors for the development of diabetic nephropathy include uncontrolled blood pressure, dyslipidemia, smoking, dietary protein intake, and family history. ${ }^{1}$ One study demonstrated that a scoring system, Screening for Occult Renal Disease (SCORED), weighted toward these common variables associated with CKD, may be a useful tool in the identification of individuals with a high likelihood of kidney disease. ${ }^{6}$

\section{Corresponding author: Keri DePatis, PharmD}

Palm Beach Atlantic University, Gregory School of Pharmacy, West Palm Beach, FL 33403

Email: kdepatis31@midwestern.edu
According to the United States Renal Data System, total Medicare spending on both chronic kidney disease (CKD) and end-stage renal disease (ESRD) is over \$114 billion annually. ${ }^{2}$ Increased screening among high-risk patients is necessary to identify the early stages of CKD, potentially resulting in earlier treatment, slower progression, fewer complications, and decreased healthcare expenditures for the disease.

Pharmacists are well-positioned to assume a more active role in screening for CKD in the primary care setting to improve patient care, particularly in these high-risk patient populations. Studies have investigated pharmacist screening for other major diseases such as osteoporosis, cardiovascular disease, depression, hypercholesterolemia, and peripheral vascular disease, indicating the feasibility of pharmacist-delivered screening services. ${ }^{7}$

One method used by pharmacists to encourage adherence amongst their patients is motivational interviewing (MI). $\mathrm{Ml}$ is a patient-centered, directive approach for enhancing motivation to change by assessing a patient's knowledge and exploring, as well as resolving ambivalence. ${ }^{8} \mathrm{MI}$ has previously been shown to be effective in smoking cessation, human immunodeficiency virus (HIV) risk reduction, weight loss, decreased alcohol use, 
and cholesterol level control. 9,10,11 Miller, et al. concluded that $\mathrm{MI}$ also shows promise for increasing patient willingness to undergo health screenings. ${ }^{12}$

Currently, there is no published literature evaluating the effectiveness of $\mathrm{MI}$ in increasing patient follow-ups with CKD screening. Given the opportunities for pharmacist-delivered screening services and the potential for $\mathrm{Ml}$ to increase a patient's willingness to undergo health screenings, this study sought to determine the impact of pharmacist-delivered $\mathrm{MI}$ versus typical education (TE) methods on the rates of microalbuminuria screening in diabetic patients of low socioeconomic status at high-risk for CKD.

\section{Methods}

This was a prospective, randomized, community intervention pilot study conducted at the Community Health Center $(\mathrm{CHC})$ of West Palm Beach. The research protocol was approved by the Palm Beach Atlantic University Institutional Review Board. Pharmacists at the $\mathrm{CHC}$ provide chronic disease state management services for diabetes mellitus, hypertension, and dyslipidemia through a collaborative practice agreement for patients of low socioeconomic status who are uninsured and do not have access to healthcare services.

From October 2018 to February 2019, pharmacists and student pharmacists screened patients with diabetes within their clinic to identify patients at high-risk for CKD, indicated by a score of 4 or greater on the validated SCORED tool (see Figure 1). ${ }^{5}$ Per Bang, et al., patients with a score of 4 or greater on the SCORED tool have a $20 \%$ chance of having CKD. ${ }^{6}$ Patients that scored less than 4 on the SCORED tool and patients with a diagnosis of CKD or ESRD, per patient-report or as documented in the electronic medical record, were excluded from the study. High-risk patients were randomized to one of four groups to receive faceto-face education sessions from a pharmacist or student pharmacist: 1) one TE session, 2) two TE sessions, 3) one MI session, or 4) two $\mathrm{MI}$ sessions. The TE or $\mathrm{MI}$ session was incorporated into the regular clinic visit and typically took 2 to 5 minutes to complete. Scripts followed during the TE and MI education sessions are provided in Appendix A. After education was provided, patients were given the option to undergo urine testing with a urine dipstick to detect albumin/creatinine ratio, which was provided at no cost to the patients. An albumin/creatinine ratio $\geq 30 \mathrm{mg} / \mathrm{dL}$ was considered positive. The urine dipstick used had a sensitivity of $96.5 \%$ and specificity of $98.3 \%$ for the microalbumin test.

The primary outcome of this study was the rate of urinary albumin testing. The secondary outcome was the rate of angiotensin-converting enzyme inhibitor (ACE-I) or angiotensin II receptor blocker (ARB) initiation, which have been found to reduce the progression of $C K D$, in patients found to have albuminuria who were not currently prescribed either an ACE-I or ARB. Medication adherence was assessed in patients with albuminuria who were currently prescribed either an ACE-I or ARB by calling the patients pharmacy to review refill history or with pill counts for patients who obtained their ACE-I/ARB from clinic supplies.

Statistical analysis was done using Microsoft Excel ${ }^{\circledR}$. Differences in the primary and secondary outcomes were assessed for significance using Fisher's exact test. To achieve a power of $82 \%$, it was calculated that a sample of 37 patients per intervention type would be needed. This is assuming a $5 \%$ alpha error level with a $25 \%$ response difference between intervention types.

Figure 1. Questionnaire for risk evaluation and potential screening adopted from Bang, et al. ${ }^{6}$

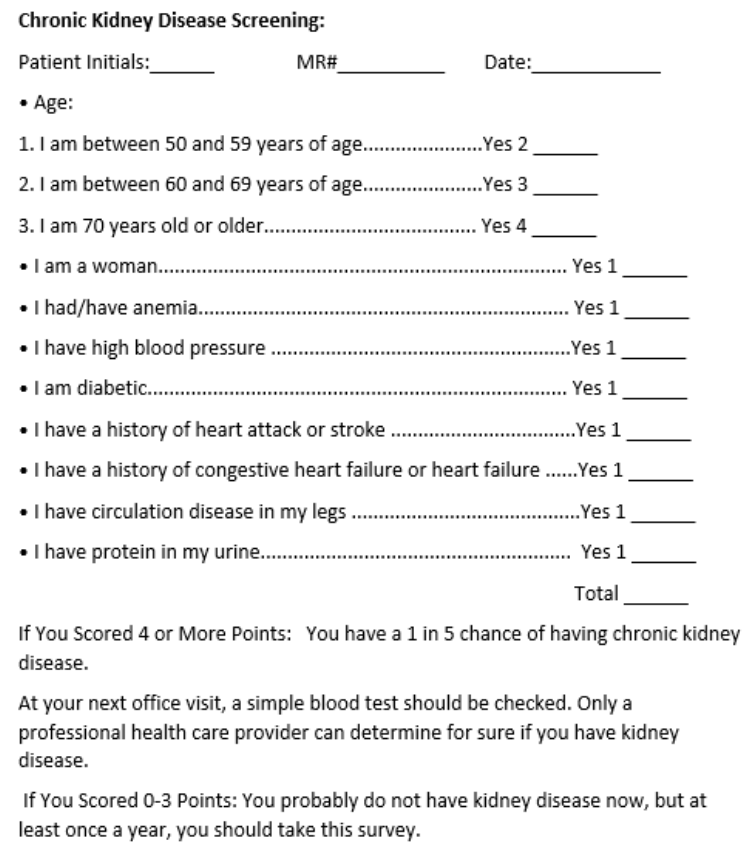


Results

Table 1: Baseline Characteristics

\begin{tabular}{|l|c|c|c|}
\hline \multicolumn{1}{|c|}{ Variable } & $T E$ & $M I$ & Overall \\
\hline $\mathrm{N}$ & 15 & 15 & 30 \\
\hline Mean age, years & 62.6 & 59.1 & 60.8 \\
\hline Female, $\mathrm{n}(\%)$ & $11(73)$ & $10(67)$ & $3(70)$ \\
\hline Anemia & $0(0)$ & $3(20)$ & $28(93)$ \\
\hline Hypertension, $\mathrm{n}(\%)$ & $13(87)$ & $15(100)$ & $4(13)$ \\
\hline Clinical ASCVD, $\mathrm{n}(\%)$ & $2(13)$ & $2(13)$ & $4(13)$ \\
\hline Heart Failure, $\mathrm{n}(\%)$ & $1(7)$ & $3(20)$ & $1(3)$ \\
\hline Circulation Disease, $\mathrm{n}(\%)$ & $0(0)$ & $1(7)$ & $0(0)$ \\
\hline Proteinuria, $\mathrm{n}(\%)$ & $0(0)$ & $0(0)$ & 5.6 \\
\hline Risk Score, avg & 5.5 & 5.7 & $24(80)$ \\
\hline Currently prescribed an ACE-I/ARB, $\mathrm{n}(\%)$ & $11(73)$ & $13(87)$ & \\
\hline
\end{tabular}

Table 2: Primary and Secondary Outcomes

\begin{tabular}{|c|c|c|c|c|}
\hline Outcome & $T E$ & $M I$ & Overall & P-value \\
\hline Urinary albumin screening, $\mathrm{n} /$ total $\mathrm{n}(\%)$ & $13 / 15(87)$ & $15 / 15(100)$ & $28 / 30(93)$ & 0.4828 \\
\hline ACE-I/ARB initiation, $n /$ total $n(\%)$ & $2 / 2(100)$ & $1 / 2(50)$ & $3 / 4(75)$ & 1.000 \\
\hline Proteinuria, $\mathrm{n} /$ total $\mathrm{n}$ of screened patients (\%) & $7 / 13(54)$ & $8 / 15(53)$ & $15 / 28(54)$ & - \\
\hline
\end{tabular}

From October 2018 to February 2019, a total of 30 patients at high risk for CKD were randomized to one of four groups to receive either one or two face-to-face education sessions from a pharmacist or student pharmacist using either MI or TE methods. Given that all patients in groups 2 or 4 accepted the screening after the first MI or TE session and therefore, did not require a second session, analysis was done for the first session only.

The self-reported characteristics of the patients at baseline based on the SCORED tool were well-balanced among study groups (Table 1). The mean age among all patients was 60.8 years, and $70 \%$ of the patients were women. The average risk score among all patients was 5.6. Age, female sex, and concomitant hypertension were the most common risk factors resulting in a score of 4 or greater on the SCORED tool. Concomitant hypertension was present in 13 of patients in the TE group, 11 of which were prescribed an ACE-I or ARB prior to the study. All patients in the MI group had a diagnosis of hypertension, 13 of which were prescribed an ACE-I or ARB prior to randomization. Adherence to the previously prescribed ACE-I or ARB was found in $85 \%$ of patients in MI group and $91 \%$ of patients in TE group.

There was no significant difference in the rate of urinary albumin screening ( $87 \%$ in TE vs. $100 \%$ in $\mathrm{MI}, \mathrm{P}=0.4828$ ) between education groups (Table 2). Among the patients screened, 15 (54\%) of patient were found to have proteinuria.

Education method also did not have a significant impact on the rate of ACE-I or ARB initiation ( 2 of 2 patients in TE and 1 of 2 patients in $\mathrm{MI}, \mathrm{P}=1.000$ ) in patients found to have proteinuria that were not currently prescribed an ACE-I or ARB (Table 2).
Of the three patients that accepted ACE-I or ARB initiation, two patients were found to be non-adherent to the newly prescribed medication.

\section{Discussion}

The preceding results suggest that pharmacist-delivered microalbuminuria screening results in clinically meaningful proteinuria identification in diabetic patients of low socioeconomic status at high-risk for CKD. In our study, $54 \%$ of patients screened were positive for albuminuria, despite no known history of proteinuria, which is substantially higher than the projected $20 \%$ risk of CKD with a "high-risk" score of 4 or greater per Bang et al. ${ }^{6}$ Vart et al. suggests that patients of low socioeconomic status are at increased risk of CKD complicated by cardiovascular events and significant decline in renal function compared to patients with traditional risk factors alone, which may account for the relatively high proteinuria rates in our patient population. ${ }^{13}$ In traditional patient populations, population-based screening for albuminuria may result in fewer cardiovascular events, cardiovascular deaths, and dialysis cases in a cost-effective manner. ${ }^{14}$ The intervention used in this study represents a possible strategy to increase screening rates in all patient populations, as it required a relatively low investment by the pharmacist in terms of time and cost of the urine test strips. Each intervention took less than five minutes per patient and the urine test strips were approximately $\$ 2$ each. This efficiency in terms of cost and time is particularly important in the indigent care setting where resources are limited, but may also be used for screenings in other outpatient settings.

Similar to the results seen in Chang et al., our study emphasizes that pharmacist intervention may result in increased urinary 
albumin screening rates in patients previously unscreened. ${ }^{15}$ The results of this study indicate that $\mathrm{MI}$ had no impact on rates of urinary screening or ACE-I/ARB initiation compared to TE methods. Therefore, while pharmacist intervention may increase compliance with routine screening recommendations, it seems that the manner in which the screening services are offered has no significant impact on patient willingness to undergo screenings.

Although a majority of patients agreed to urinary screening, non-adherence to newly prescribed ACE-I/ARB therapy in response to proteinuria remained high. The reason for nonadherence is not known, but could be due to low patient health literacy and/or transportation difficulties, which are common barriers to care in the indigent setting. Although there was no significant difference between $\mathrm{MI}$ and $\mathrm{TE}$ in regards to acceptance of screening, MI may still have a role in improving medication compliance.

Although the pragmatic study design indicates feasibility of the intervention in the primary care setting, this study had some important limitations. Because of the small sample size, power was not met, resulting in a possible type II error. Furthermore, urinary albumin excretion may be elevated in certain conditions (e.g. infection, fever, marked hyperglycemia/hypertension), and current guidelines recommend two of three abnormal measurements over several months to confirm a diagnosis of albuminuria. ${ }^{3}$ Given the time and resource constraints of our practice site, this was not done, and the accuracy of proteinuria found in these high-risk patients may be questionable. In patients with suboptimal adherence and follow-up, such as those in indigent care settings, providers be hesitant to initiate ACE-I/ARB therapy due to safety concerns. In our patient population, all patients started on an ACE-I/ARB as a part of the study had follow-up labs done within 4 to 6 weeks after therapy initiation.

Patient care at the $\mathrm{CHC}$ relies heavily on the involvement of student pharmacists, which may have affected data collection and the consistency of the intervention delivered. While the pharmacists that delivered the intervention were formally trained in $\mathrm{Ml}$, the student pharmacists were not. In an attempt to reduce the impact of this on the results, a topic discussion on MI was done with all student pharmacists, and a protocol and scripts were developed to standardize the education interventions. Given all of the patients in the MI group accepted the screening after the first session, differences in MI training likely did not contribute to the lack of statistical significance observed.

This study was done in an uninsured, low-income patient population, and the intervention was done at no cost to the patient, possibly increasing patient participation. Acceptance of microalbuminuria screening may be lower in patients that must pay for screening in an alternative outpatient setting. Furthermore, because lisinopril and losartan are on the free or low-cost lists at a supermarket chain in Florida, a majority of our low-income population were prescribed an ACE-I/ARB prior to randomization. In other patient populations, fewer patients without previously identified proteinuria, particularly those with concomitant hypertension that are self-identified black, may have lower rates of current ACE-I/ARB use. Therefore, a greater impact on ACE-I/ARB initiation may be found in those patients. Finally, because the urine test strips were read visually, differences in color perception may have overestimated the level of proteinuria. However, this likely did not have had a profound impact on patient outcomes given the high rates of concomitant hypertension and ACE-I/ARB use in our patient population prior to randomization.

In conclusion, while it appears that $\mathrm{MI}$ does not impact patient acceptance rates of microalbuminuria screening and ACE-I/ARB initiation, this study demonstrates the feasibility of pharmacistdelivered microalbuminuria screening in patients at high-risk for CKD in the outpatient setting. Use of the SCORED tool and urine albumin test strips resulted in the identification of proteinuria in $54 \%$ of screened patients that had not been previously diagnosed with CKD. In practice, this may be a costeffective method for increasing outpatient microalbuminuria screening in high-risk patients. Future studies should measure the effect of these educational methods on factors affecting CKD progression, such as continued medication adherence and limitation of protein intake, and assess the impact of pharmacist involvement on other clinical outcomes, including glycemic and blood pressure control, drug optimization to decrease albuminuria, and medication compliance.

Conflicts of Interest: We declare no conflicts of interest or financial interests that the authors or members of their immediate families have in any product or service discussed in the manuscript, including grants (pending or received), employment, gifts, stock holdings or options, honoraria, consultancies, expert testimony, patents and royalties.

Treatment of Human Subjects: IRB review/approval required and obtained

\section{References}

1. Gross JL, de Azevedo MJ, Silveiro SP, et al. Diabetic nephropathy: Diagnosis, prevention, and treatment. Diabetes Care 2005;28(1):164-176.

2. United States Renal Data System. 2018 Annual Data Report. https://www.usrds.org/adr.aspx (accessed Dec 11, 2018).

3. American Diabetes Association. Microvascular complications and foot care: Standards of medical care in diabetes - 2019. Diabetes Care 2019 Jan; 42(Supplement 1): S124-S138.

4. Mainous AG III, Gill JM. The lack of screening for diabetic nephropathy: Evidence from a privately insured population. Fam Med 2001;33:115-119.

5. Byun SH, Ma SH, Jun JK, et al. Screening for diabetic retinopathy and nephropathy in patients with diabetes: a nationwide survey in Korea. PLoS One 2013;8(5):e62991. 
6. Bang $\mathrm{H}$, Vupputuri S, Shoham DA, et al. Screening for occult renal disease: A simple prediction model for chronic kidney disease. Arch Intern Med 2007;167:374-381.

7. Ayorinde AA, Porteous T, Sharma P. Screening for major diseases in community pharmacies: a systematic review. Int J Pharm Pract 2013;21(6):349361.

8. Miller WR, Rollnick S. Motivational interviewing: preparing people for change. New York: Guilford; 2002.

9. Dunn C, Deroo L, Rivara FP. The use of brief interventions adapted from motivational interviewing across behavioral domains: a systematic review. Addiction 2001;96:1725-42.

10. Burke BL, Arkowitz H, Menchola M. The efficacy of motivational interviewing: a meta-analysis of controlled clinical trials. J Consult Clin Psychol 2003;71:843-61.

11. Rubak S, Sandbaek A, Lauritzen T, Christensen B. Motivational interviewing: a systematic review and meta-analysis. Br J Gen Pract 2005;55:305-12.

12. Miller SJ, Foran-Tuller K, Ledergerber J, et al. Motivational interviewing to improve health screening uptake: A systematic review. Patient Educ Couns. 2017;100(2):190-198.

13. Vart $P$, Reijneveld $S A$, Bultmann $U$, et al. Added value for CKD among the elderly or persons with low socioeconomic status. Clin J Am Soc Nephrol. 2015;10:562-570.

14. Boersma C, Gansevoort RT, Pechlivanoglou P, et al. Screen-and-treat strategies for albuminuria to prevent cardiovascular and renal disease: costeffectiveness of nationwide and targeted interventions based on analysis of cohort data from the Netherlands. Clin Ther. 2010;32(6):1103-21.

15. Chang $A R$, Evans $M$, Yule $C$, et al. Using pharmacists to improve risk stratification and management of stage 3A chronic kidney disease: a feasibility study. BMC Nephrol. 2016;17:168. 


\section{Appendix A}

\section{Typical Education Session}

1. If previously identified as 'high-risk', inform patient and ask permission to discuss CKD screening.

2. Provide patient education using the following information:

a. Because you have diabetes and/or hypertension, you have a higher risk of kidney damage.

b. Most people do not know that they have damage to their kidneys until it is too late, so it is important to get screened, so you can prevent the damage from getting worse.

c. One of the ways we can tell if you have kidney damage is to test your urine to see if it has protein in it.

d. If there is protein found in your urine, there is a medication we can start that can help prevent it from getting worse.

e. At the clinic, we provide this testing free. Would you be interested in getting it done today?

\section{Motivational Interviewing Session}

1. Ask permission to explore CKD screening.

2. Provide patient education using the following motivational interviewing technique:

a. Ask patient what they know about kidney disease and diabetes.

b. Use reflective listening

c. Summarize

d. Provide-Elicit-Provide: Ask if it would be okay to share some additional information about screening and ways to prevent or reduce the progression of CKD with them? If they respond "yes", provide information and then make sure to elicit feedback after providing. If they say "no", hold off.

i. Because you have diabetes and/or hypertension, you have a higher risk of damaging your kidneys.

ii. Most people do not know that they have damage to their kidneys until it is too late, so it is important to get screened, so you can prevent the damage from getting worse.

iii. One of the ways we can tell if you have kidney damage is to test your urine to see if it has protein in it.

iv. If there is protein found in your urine, there is a medication we can start that can help prevent it from getting worse.

e. Assess motivation, confidence, and readiness to get screened by asking, "We provide the screening test free of charge here at the clinic. How would you feel about getting screened today?"

f. Explore ambivalence.

i. Ask what are some reasons you think you may want to be screened?

ii. Ask what are some reasons you think you may NOT want to be screened?

g. Ask: What would you like to do next?

h. Make a plan with patient for the future (i.e. provide urine sample today, at later date, etc.) 